PAPER

\title{
Surgical management of tuberculum sellae meningiomas: involvement of the optic canal and visual outcome
}

\author{
U Schick, W Hassler
}

J Neurol Neurosurg Psychiatry 2005;76:977-983. doi: 10.1136/jnnp.2004.039974

See end of article for authors' affiliations .....................

Correspondence to: Dr Uta Schick, Clinic of Neurological Surgery, Wedau Kliniken, Zu den Rehwiesen 9, 47055 Duisburg, Germany; Uta_Schick@web.de

Received 26 February 2004 In revised form

13 September 2004

Accepted 28 October 2004
Objective: To present a large series of surgically treated tuberculum sellae meningiomas with particular regard to involvement of the optic canal and visual outcome.

Methods: A retrospective analysis was done on 53 patients (40 female) with meningiomas originating from the tuberculum sellae who underwent surgery between 1991 and 2002. The standard surgical approach consisted of pterional craniotomy. Sixteen meningiomas extended posteriorly onto the diaphragma sella, 29 anteriorly to the planum sphenoidale, and 19 to the anterior clinoid process. Thirty seven tumours involved the optic canal, three bilaterally. Follow up ranged from 6 to 108 months (mean 29.9 months).

Results: Total macroscopic resection was achieved in 48 patients. Median tumour size was $2.6 \mathrm{~cm}$. Postoperatively, visual acuity improved in 20 patients and deteriorated in seven. Preoperative and postoperative visual acuity worsened with increasing duration of preoperative symptoms and with increasing age. Extension into the intraconal space was a negative predictor. However, tumour size did not influence visual acuity. Recurrence occurred in two cases (21 and 69 months postoperatively). Two patients died from causes unrelated to the tumour.

Conclusions: In the majority of patients with tuberculum sellae meningiomas, total resection may be achieved through a pterional approach with minimal complications.
M eningiomas of the anterior skull base account for $40 \%$ of all intracranial meningiomas. ${ }^{1}$ Of these, about $25 \%$ are tuberculum sellae tumours. Women are affected three times more often than men. The disease is diagnosed in the fourth or fifth decade. ${ }^{2}$ The tumour originates from the tuberculum sellae, chiasmatic sulcus and limbus sphenoidale, and the diaphragma sellae. ${ }^{13}$ Tuberculum sellae meningiomas very commonly extend into both optic canals, a problem that is underemphasised in published reports. ${ }^{1}$ Visual loss in one eye with optic atrophy is the initial and most common symptom. These tumours tend to displace the optic chiasm backwards and the optic nerves laterally and superiorly. Compression of the chiasma is typically accompanied by bitemporal hemianopia. ${ }^{14}$ Different techniques for resection include the pterional approach ${ }^{56}$ or a unilateral or bilateral subfrontal approach. ${ }^{4} 7$ Indispensable surrounding structures such as the optic nerves and chiasm, pituitary stalk, and anterior choroidal artery must be respected. ${ }^{4}$

This report summarises a retrospective analysis of 53 tuberculum sellae meningiomas treated at the neurosurgical clinic of Duisburg over an 11 year period. The study places special emphasis on surgical technique and visual outcome. Postoperative visual outcome is analysed and related to preoperative visual loss, tumour volume and topography, and to the age of the patients. Prognostic variables for vision are defined, and compression patterns of the optic nerve and chiasm elaborated. The frequency and extension of optic canal involvement and its possible negative effect on prognosis is investigated and the importance of optic canal inspection for possible tumour extension discussed.

\section{METHODS}

\section{Patient characteristics}

The series consisted of 53 patients with tuberculum sellae meningiomas, all of whom underwent surgery from 1991 to 2002. The cohort comprised 40 women and 13 men (female to male ratio, 3:1). Follow up data were available from 6 to 108 months, with a median of 29.9 months. The mean (SD) age of the patients was 52.6 (13.6) years (range 27 to 78 ).

Ophthalmological examination consisted of testing the patients' visual acuity (Snellen notation), fundoscopy, and Goldmann perimetry for visual field defects. A change of $\geqslant 2$ lines indicated improvement or deterioration. Oculomotor function was evaluated, along with other neurological functions. Endocrinological tests (adrenal, thyroid, and gonadal axes, specific gravity of the urine, fluid balance) were done preoperatively and one week and three months postoperatively.

All patients underwent evaluation by computed tomography (CT) or magnetic resonance imaging (MRI) with and without contrast agent. High resolution CT showed hyperostosis of the planum sphenoidale and tuberculum sellae or calcification within the tumour. Both $\mathrm{Tl}$ and $\mathrm{T} 2$ weighted MRI were done in three planes to analyse the relation to vascular and neighbouring structures. Follow up MRI was scheduled at three and 12 months postoperatively, and then annually.

Information on clinical history, signs, surgical approach, and outcome was obtained retrospectively by review of the patient's case notes and the radiological reports.

All 53 meningiomas originated from the tuberculum sellae. Sixteen extended posteriorly into the diaphragmal region, 29 anteriorly to the planum sphenoidale, and 19 to the anterior clinoid process. Thirty seven involved the optic canal (fig 1). Nine extended along the optic canal into the intraconal orbital compartment. One presented as a large bulbiform orbital mass; this patient presented with one large mass at the tuberculum sellae and the second mass around the optic nerve. It is probable that this intraorbital tumour had extended into the intracranial space, forming a second tumour nodule. The size of the tumour varied from $1 \mathrm{~cm}$ to $5 \mathrm{~cm}$, with a median diameter of $2.6 \mathrm{~cm}$. 

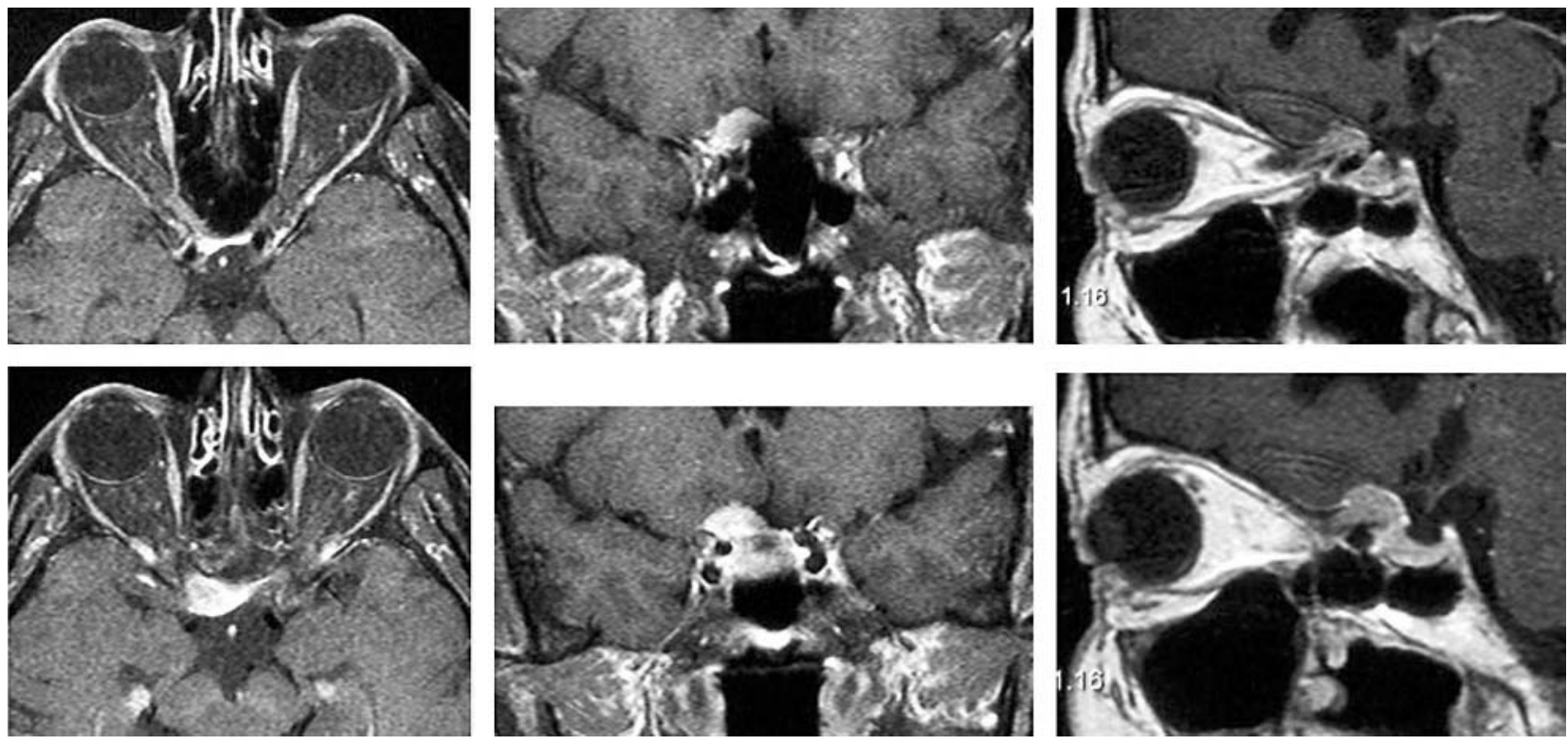

Figure 1 Post-contrast T1 weighted magnetic resonance imaging (transverse, fat suppressed, coronal water excited, sagittal only enhanced) showing extension of the tuberculum sellae meningioma into the right optic canal.

\section{Surgical technique}

All operations were carried out through a unilateral frontotemporal approach, on the side of visual deterioration. In bilateral involvement the right side was preferred. This pterional approach is well described. ${ }^{89}$

Drainage of cerebrospinal fluid (CSF) was done by lumbar drainage and later by opening the basal cisterns. The sylvian fissure was routinely opened and the Ml segment of the middle cerebral artery exposed (fig 2, step 1). The internal carotid artery was identified (step 2) and this led to the ipsilateral optic nerve ( step 3), which might be covered by tumour. The anterior tumour capsule was opened and the basal blood supply was interrupted by lifting the tumour and coagulating the feeding arteries (step 4) until the ipsilateral optic nerve appeared. The tumour was further debulked to reduce its volume until the contralateral optic nerve became visible ( step 5). The tumour located medial of the ipsilateral optic nerve was then removed from the skull base (step 6). This step was followed by dissection of the arachnoidal plane from the ipsilateral optic nerve (step 7). After interruption of the basal blood supply, the tumour became soft and anaemic and thus could easily be detached from the arachnoidea of the gyrus rectus, the $\mathrm{Al}$ segment of the anterior cerebral artery, or the anterior communicating artery. Finally, the tumour was removed from the Al segment (step 8) and the chiasm (step 9).

Protection of the optic and chiasmatic blood supply is extremely important to preserve vision. In particular, the small vessels from the carotid artery to the optic nerve have to remain in their arachnoidal layer and should not be occluded. In cases of hyperostotic planum sphenoidale the basal dura was excised and the bone was drilled away. The defect was covered with fat, freeze dried dura, and fibrin glue. In optic canal involvement the first $3-5 \mathrm{~mm}$ of the optic canal, which are fibrous, were opened. The bony roof was drilled away if there was further tumour extension. The ophthalmic artery below the optic nerve was exposed and preserved. If necessary, the optic nerve sheath was opened until the annulus of Zinn was reached and the tumour around the optic nerve was carefully removed. In contrast to optic nerve sheath meningiomas, tumour mass in the optic canal in tuberculum sellae meningiomas could easily be dissected from the optic nerve.

All operations were done by the senior author, WH. The technique has only slightly changed over the years. The most important steps remain the interruption of the basal blood supply and hollowing of the tumour. The most important structure to be exposed is the contralateral optic nerve to obtain an anatomical overview. Close to the optic nerves, no active coagulation was used and the tumour was sucked within its arachnoidal plane. In recurrent tumour growth no radical resection was done. Deroofing of the optic canal was carried out whenever intracanalicular tumour growth was suspected. The tumour is always located in the basal part of the canal. With deroofing of the canal, the optic nerve can be mobilised and then the tumour below the nerve can be sucked away. This procedure prevents recurrent tumour growth.

\section{Statistical analysis}

Spearman's correlation coefficients $(r)$ were calculated. Nonparametric testing was used because abnormal distributions were suggested by Kolmogorov-Smirnov normality tests. The non-parametric Mann-Whitney $U$ test was applied to independent samples. For two dependent samples we used the Wilcoxon test, and for more than two independent samples the Kruskal-Wallis H test. For comparisons between nominal characteristics we used $\chi^{2}$ tests. Probability values below 0.05 indicated significant differences.

\section{RESULTS}

\section{Signs and symptoms}

The mean duration of symptoms was 21.8 months (range 1 to 240 ). The preoperative duration of symptoms correlated negatively with preoperative visual acuity (Spearman's correlation coefficient $r=-0.34, \mathrm{p}=0.006)$. Postoperative recovery of visual deficits was worse within the group of patients with deficits lasting longer than six months (Kruskal-Wallis test, $\mathrm{p}=0.005$ ). There was a clear correlation between age and preoperative visual disturbances (Spearman's correlation coefficient $r=-0.42, \mathrm{p}=0.0007$ ). Visual impairment was the initial symptom in 40 patients, 


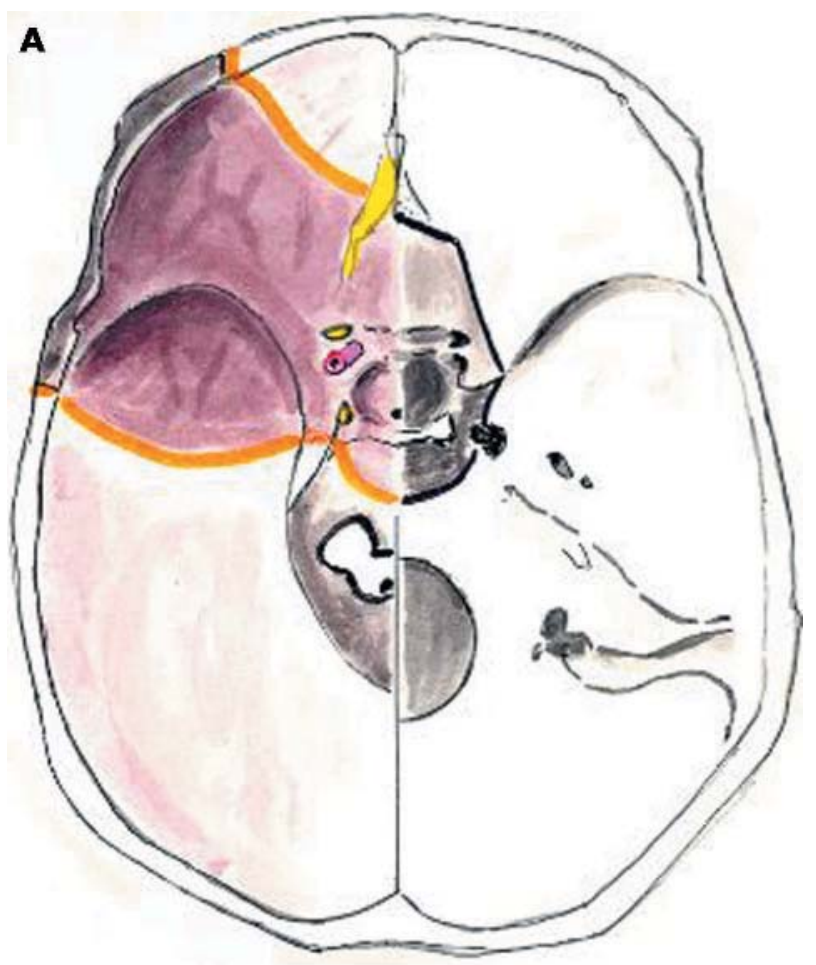

visual field defects in four, and headache in three. Six meningiomas were an incidental finding.

On admission, seven patients had normal vision, 17 still had good vision $(\geqslant 0.5), 13$ had fair vision $(<0.5$ to $>0.1)$, and 15 had no useful vision ( $\leqslant 0.1$ to 0 ) (table 1 ). Location in the intraconal compartment was associated with worse preoperative vision (Wilcoxon test, $\mathrm{p}=0.018$ ). Other extensions from the tuberculum sellae did not influence visual acuity (Wilcoxon test, NS). Visual impairment of the other eye was present in 18 patients, among whom three had no useful vision. The tumour size correlated with contralateral visual impairment (Spearman rank correlation coefficient $r=-0.32, \mathrm{p}=0.008$ ).

Fundoscopy revealed optic disk pallor in 26 patients, of whom seven showed bilateral involvement. Disc swelling was found in four patients of whom two showed bilateral swelling. All but three patients with impaired vision also had visual field defects $(n=43)$. Prechiasmal and anterior

Table 1 Overview of preoperative visual acuity (Snellen notation), visual outcome, and follow up

\begin{tabular}{|c|c|c|c|}
\hline Patient & Preoperative & Postoperative & Follow up \\
\hline 1 & 1 & 1 & 1 \\
\hline 2 & 1 & 1 & 1 \\
\hline 3 & 1 & 1 & 1 \\
\hline 4 & 1 & 1 & 1 \\
\hline 5 & 1 & 1 & 1 \\
\hline 6 & 1 & 1 & 1 \\
\hline 7 & 1 & 1 & 0.8 \\
\hline 8 & 0.8 & 0.8 & 0.9 \\
\hline 9 & 0.7 & 1 & 1 \\
\hline 10 & 0.7 & 1 & 1 \\
\hline 11 & 0.7 & 1 & 1 \\
\hline 12 & 0.7 & 1 & 1 \\
\hline 13 & 0.7 & 0.6 & 0.8 \\
\hline 14 & 0.7 & 0.4 & 0.4 \\
\hline 15 & 0.6 & 1 & 1 \\
\hline 16 & 0.6 & 0.8 & 0.8 \\
\hline 17 & 0.6 & 0.6 & 0.6 \\
\hline 18 & 0.6 & 0.6 & 0.6 \\
\hline 19 & 0.5 & 1 & 0.6 \\
\hline 20 & 0.5 & 0.5 & 0.5 \\
\hline 21 & 0.5 & 0.5 & 0.5 \\
\hline 22 & 0.5 & 0.3 & 0.3 \\
\hline 23 & 0.5 & 0.25 & 0.02 \\
\hline 24 & 0.5 & 0.1 & 0.1 \\
\hline 25 & 0.4 & 0.8 & 1 \\
\hline 26 & 0.4 & 0.8 & 0.9 \\
\hline 27 & 0.4 & 0.4 & 0.5 \\
\hline 28 & 0.3 & 0.8 & 1 \\
\hline 29 & 0.3 & 0.8 & 0.8 \\
\hline 30 & 0.3 & 0.3 & 1 \\
\hline 31 & 0.3 & 0.1 & 0 \\
\hline 32 & 0.3 & 0 & 0 \\
\hline 33 & 0.2 & 1 & 1 \\
\hline 34 & 0.2 & 0.4 & 0.6 \\
\hline 35 & 0.2 & 0.2 & 0.3 \\
\hline 36 & 0.2 & 0.2 & 0.1 \\
\hline 37 & 0.2 & 0.1 & 0 \\
\hline 38 & 0.1 & 1 & 1 \\
\hline 39 & 0.1 & 0.6 & 0.8 \\
\hline 40 & 0.1 & 0.5 & 1.2 \\
\hline 41 & 0.1 & 0.5 & 0.6 \\
\hline 42 & 0.1 & 0.5 & 0.5 \\
\hline 43 & 0.1 & 0.4 & 1 \\
\hline 44 & 0.1 & 0.3 & 0.3 \\
\hline 45 & 0.1 & 0.1 & 0.1 \\
\hline 46 & 0.05 & 0.7 & 0.7 \\
\hline 47 & 0.05 & 0.05 & 0.2 \\
\hline 48 & 0.05 & 0 & 0 \\
\hline 49 & 0 & Died & \\
\hline 50 & 0 & 0 & Died \\
\hline 51 & 0 & 0 & 0 \\
\hline 52 & 0 & 0 & 0 \\
\hline 53 & 0 & 0 & 0 \\
\hline
\end{tabular}


chiasmal compression resulted in central scotoma and temporal anopia in one eye and incomplete temporal anopia in the other eye (type $1, n=4$ ). Compression of the chiasm could provoke the classical bitemporal hemianopia (type 2) with reduction in central visual acuity in one or both eyes $(n=17$, eight complete, nine incomplete). Involvement of the intracranial optic nerve at the junction to the chiasm led to the anterior junction syndrome ${ }^{10}$ (type 3 ) with ipsilateral central scotomas and contralateral temporal anopia $(n=5)$. Lesions of the optic tract resulted in incongruent homonymous hemianopia (type 4) in three patients. Three patients had irregular small unclassified visual field defects and 11 were blind in one eye. Hyposmia was present in four patients.

Tumour related endocrinological abnormalities were seen in four patients (one had a raised prolactin level, one had polydipsia, one had partial insufficiency of the adrenal axis, and one had a raised cortisol level). The patient with central Cushing's syndrome was first operated on for the tuberculum sellae meningioma and three months later an ACTH producing intrasellar microadenoma was removed through a trans-sphenoidal approach (fig 3).

\section{General history}

Three patients suffered from multiple intracranial or spinal meningiomas, and two from optic nerve sheath meningiomas. Three patients had undergone operation for tuberculum sellae meningiomas in other neurosurgical departments $(2,7$, and 24 years before) and were referred to our centre for reoperation. One patient was misdiagnosed as a pituitary adenoma and first operated on by a trans-sphenoidal approach.

\section{Surgery}

The standard approach was the intradural pterional approach. Thirty six patients were operated on through a right sided approach and 17 through a left sided approach. The arachnoid remained intact in 47 cases. Tumour size influenced the preservation of the arachnoid ( $U$ test, $p=0.017$ ). With respect to preoperative and postoperative visual acuity, there was no difference between the groups with preserved and non-preserved arachnoid (U test, NS). However, there was a tendency to worse postoperative visual acuity in the group with non-preserved arachnoid medial to the optic nerve. The pituitary stalk could be preserved in all cases. Total macroscopic resection was achieved in 48 patients. Five patients had subtotal resection owing to small tumour rests around the vessels or the optic nerve or to bone involvement. The extent of resection did not depend on tumour size (U test, NS). The ipsilateral optic canal was explored in 37 patients. The dural part was involved in 21 cases. The bony roof was drilled away in 16 patients who had further tumour extension. Bilateral involvement of the optic canal was seen in three patients. However, the extent to which the optic canal was opened did not influence postoperative visual acuity (Kruskal-Wallis test, NS). Only tumour size was associated with the extent of canal involvement (Kruskal-Wallis test, $p=0.03$ ). However, only deroofing of the optic canal allows total tumour removal below the optic nerve within the optic canal.

Neither the extent of resection (U test, NS) nor the size of the tumour (Spearman rank correlation, NS) influenced postoperative visual acuity.

\section{Complications}

Two patients (aged 77 and 78 years) died one month postoperatively from cardioembolic brain stem infarction and septicaemia (overall mortality 3.7\%). One patient suffered a cardioembolic left occipital infarct. One epidural haematoma required reoperation and two subdural hygromas
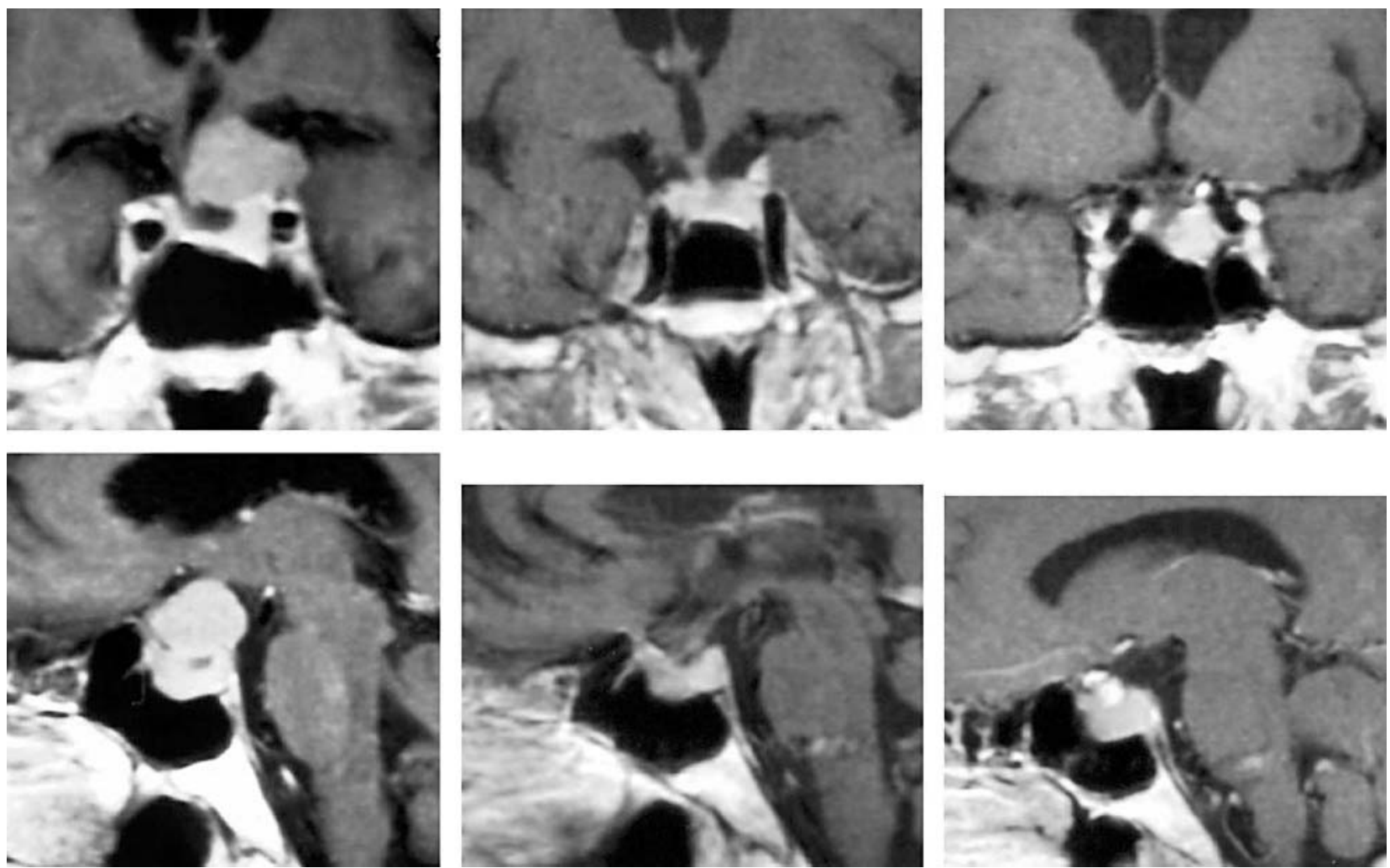

Figure 3 Coincidence of a pituitary adenoma with ACTH secretion and a tuberculum sellae meningioma. Coronal and sagittal preoperative enhanced magnetic resonance imaging (MRI) with a large suprasellar meningioma (left); postoperative MRI scans after removal of the tuberculum sellae meningioma through a left pterional approach (middle); and MRI three months later before trans-sphenoidal approach to left sided intrasellar pituitary adenoma (right). 
were punctured. One patient had to undergo a second operation for rhinoliquorrhea from the frontal sinus. Five patients had a temporary cerebrospinal fluid fistula and were adequately treated by lumbar drainage. Four suffered from an epileptic seizure in the first postoperative week. Secondary visual loss in the right eye occurred in one patient on the 12th postoperative day; he underwent treatment with nimodipine and haemodilution therapy, with complete recovery within five days. One patient received hydrocortisone as replacement therapy for three months. Four patients developed permanent hyposmia and one developed anosmia.

\section{Outcome}

On discharge, the absolute postoperative visual acuity improved in 20 patients ( $\geqslant 2$ lines in Snellen notation), remained unchanged in 25, and deteriorated in seven. Preoperative vision correlated highly with postoperative vision (Spearman's correlation coefficient $r=0.69$, $\left.\mathrm{p}=4.9 \times 10^{-9}\right)$. Age was negatively correlated with the postoperative visual acuity (Spearman's correlation coefficient $r=-0.34, p=0.006$ ). Longer duration of preoperative symptoms correlated with worse recovery (Spearman's correlation coefficient $\left.r=-0.47, \mathrm{p}=1.85 \times 10^{-4}\right)$. Deterioration of vision was not associated with involvement of the optic canal. In these cases, there was a tendency to interruption of the arachnoidal plane. Thus deterioration of visual acuity seems to be a vascular problem.

Eighteen patients with visual field defects improved markedly postoperatively. In eight patients the defects resolved completely. However, seven patients worsened. No patient developed diabetes insipidus.

\section{Follow up}

Vision generally improved rapidly within the first weeks after surgery, but continued to improve for the next year. At follow up, the absolute visual acuity improved further in four patients and worsened in four. Only one patient with bad vision $(\leqslant 0.1$ to 0 ) improved further. Patients older than 50 years had worse recovery ( $U$ test, $p=0.005$ ). Vision did not deteriorate with longer follow up (Spearman's correlation coefficient, NS).

\section{Recurrence}

Recurrence was found in two cases (3.7\%), 21 and 69 months postoperatively. However, follow up varied from 6 to 108 months. One of these patients became symptomatic with a new visual field defect and the other with headache. The case with early recurrence had a history of multiple meningiomas and the $4 \mathrm{~cm}$ tumour was only subtotally resected at the first operation. Both patients underwent reoperation with good results.

\section{DISCUSSION}

\section{Outcome and prognostic factors}

The major visual signs associated with tuberculum sellae meningiomas are visual loss (98\%) and optic atrophy (78\%). The Foster-Kennedy syndrome of unilateral optic atrophy and contralateral papilloedema occurs in 5\% of these patients. ${ }^{1}$ The clinical presentation is of visual loss in one eye with optic atrophy that develops into blindness and diminution of acuity in the opposite temporal visual field. ${ }^{1}$ About $80 \%$ of patients suffer from visual disturbance for a period of between three months and 20 years, with an average of more than two years. ${ }^{4}$

Hemiparesis is seen in $15 \%$ and anosmia in $11 \%$. Pituitary insufficiency has been reported to occur in up to $22 \%$ of patients. ${ }^{1}$ However, in the study by Fahlbusch and Schott ${ }^{5}$ endocrine disturbances did not play a major role, but may be a more important factor in very large tumours.
Improvement of vision was noted in 50-66\%, while in 17$28 \%$ it remained unchanged. Deterioration was noted in 10$25 \%{ }^{4}{ }^{911}$ Tumours larger than $3 \mathrm{~cm}^{1}$ or $4 \mathrm{~cm}^{2}$ are thought to be associated with increased morbidity and a lack of improvement in vision following surgery, as well as with increased mortality. ${ }^{1}$ Visual outcome was favourably affected when the tumour was less than $3 \mathrm{~cm}$, the preoperative visual loss was less than $50 \%$ and present for less than two years, and the optic discs appeared normal on fundoscopy. ${ }^{11}$ Poor prognostic factors include tumour extension into the optic foramen or the diaphragma sellae. Fahlbusch and Schott ${ }^{5}$ reported a markedly increased risk of visual deterioration (up to $40 \%$ ) in patients in whom the duration of symptoms was longer than one year.

In the series by Zevgaridis et al, ${ }^{12}$ visual prognosis was favourably affected by age under 54 years, duration of symptoms of less than seven months, and the presence of an intact arachnoid membrane around the lesion. Severe preoperative loss of visual acuity appeared to be an unfavourable prognostic factor. Severe morbidity occurred in $6.4 \%$. However, in our study tumour size and location had no effect on outcome, in contrast to some previous reports. Our findings are supported by recent studies. ${ }^{5}$

Visual recovery is sometimes possible in patients with poor preoperative visual acuity (eight of our patients in this group improved, and six even regained good vision). Postoperative severe deterioration is mostly permanent. Only one patient with secondary visual loss in one eye on the 12th postoperative day recovered completely.

The incidence of total removal of tuberculum sellae meningiomas has varied greatly, with reported rates of 40$95 \% .^{1}$ Mortality ranges from none to $7 \% .^{1}$ A large study on recurrence of cranial base meningiomas ${ }^{13}$ showed a perioperative mortality of $10.8 \%$, and $9.7 \%$ died within 10 years. Our mortality was $3.7 \%$ in our oldest patients. Cornu et $a l^{14}$ found that the general condition of the patient was more important than age itself. Patients in poor general condition had a fivefold increased risk of a poor outcome.

\section{Optic canal involvement}

Bilateral involvement of the optic canal is described. ${ }^{1}$ Growth of tumour in the optic canal is thought to be associated with worse visual prognosis. ${ }^{15}$ Arai et $a l^{3}$ emphasised that the presence of preoperative visual disturbance implies the need for optic canal deroofing. Two of 13 cases required deroofing on both sides because the tumour extended into the canal on the non-symptom side. Four of eight cases with bilateral visual disturbance required optic canal deroofing on both sides. Andrews and Wilson ${ }^{16}$ noted that patients with invasion of the optic canal show less frequent improvement in visual acuity or visual fields. DeMonte ${ }^{1}$ favours optic canal inspection at the end of the surgery. Residual tumour in the canal may be the site of recurrence or the cause of a lack of visual improvement following surgery. ${ }^{1}$

In our opinion, when the tumour is in the optic canal it should be followed and removed completely, together with any dura involved. Tumour within the optic canal can easily be removed because there are no adhesions. However, it may be difficult to dissect the tumour from the chiasm and nerves within the cistern. An intraconal tumour location had a negative effect on visual outcome. The high proportion of intracanalicular extension $(69.8 \%)$ supports our procedure of surgical inspection and tumour removal.

\section{Pathogenesis}

There are different mechanisms for preoperative optic nerve injury: ischaemia, compression, demyelination, and tumour invasion. ${ }^{17}$ Compressive mechanical injury leads to small vessel compromise and demyelination, especially in patients 
with a long duration of visual loss before surgery. Assuming that there is no additional intraoperative trauma to the optic nerve, incomplete or no recovery of visual function after surgery may imply chronic severe preoperative ischaemic or compressive damage and demyelination. Compressive injury can at least be reversed by surgery. Thus decompression of the optic pathway is the main goal of the surgery. Zevgaridis et $a^{12}$ hypothesise that ischaemia is the cause of primary visual loss rather than simply optic nerve compression. Younger patients are able to compensate for microvascular deprivation more effectively than older ones. This could explain our finding of worse vision and poor recovery in elderly patients. Andrews and Wilson ${ }^{16}$ postulated direct compression or invasion of the nerve, or preoperative damage to its blood supply. When the tumour is densely adherent to the optic apparatus, the best choice is to leave tumour fragments. Preservation of the vascular supply to the nerves is important. ${ }^{57}$ Occlusion of perforating vessels contributes to postoperative visual deterioration and should be avoided.

\section{Surgical approach}

Symon and Rosenstein ${ }^{11}$ reached the tumour subfrontally along the midline following right frontal craniotomy. AlMefty et $a l^{7}$ chose unilateral or bilateral frontal craniotomy, depending on the tumour size. Ohta $e t$ al ${ }^{4}$ used the pterional approach in 15 patients, the orbitozygomatic in 10, and the bilateral subfrontal approach in six. Arai et al describe a transcranial trans-sphenoidal approach for tuberculum sellae meningiomas. They operated on 21 patients through a bifrontal craniotomy, removal of the orbital rim, transsection of the superior sagittal sinus, dissection along the olfactory tracts and interhemispheric fissure, and drilling out of the skull base. In all cases, the optic canal and sphenoid sinus and in some cases the ethmoid sinus was opened.

In our opinion this approach is too extensive. Cerebrospinal fluid leakage occurs more often with opening of the sinus. Olfactory function, with postoperative anosmia and partial recovery in 16 of 21 patients, was worse than in other series. The pterional approach carries less risk for the olfactory nerve and requires less brain retraction. All structures can easily be reached through a pterional approach (fig 2 ). The approach is conducted from the side where the optic nerve is more severely damaged.

The differential diagnosis of a suprasellar mass mimicking a pituitary macroadenoma is sometimes a problem. In our series, we had one case where a tuberculum sellae meningioma and an ACTH producing pituitary adenoma were both present (fig 3), and one cases in which a meningiomas was misinterpreted as an adenoma and first operated on through a transnasal trans-sphenoidal approach.

\section{Recurrence}

The likelihood of recurrence is related to the extent of tumour removal. ${ }^{6}$ In the series by Raco et al, ${ }^{6}$ involving 63 patients who underwent radical tumour removal, only one recurrence was observed, whereas three of six patients with subtotal resection developed a recurrence. These data are in agreement with the findings of Symon and Rosenstein, ${ }^{11}$ with one recurrence in 70 radically removed meningiomas compared with a recurrence rate of $33.3 \%$ in 12 cases with subtotal removal. Mathiesen et al ${ }^{13}$ presented a series of 315 cranial base meningiomas with a mean follow up period of 18 years. The five year recurrence rate was $4 \%$ for patients undergoing radical surgery, and $25-45 \%$ for patients undergoing subtotal operations. Only in the series of Fahlbusch and Schott ${ }^{5}$ was the recurrence rate extremely low, at $2.1 \%$ (one of 47 cases), with complete tumour removal in $98 \%$. The worst outcome was found in medial sphenoid wing/clinoidal meningiomas and in tumours invading the cavernous sinus. In our series, one recurrence was observed in a subtotally removed tumour, and one in a totally removed tumour. The recurrence in subtotal removal occurred earlier. Complete tumour resection should not be achieved at the cost of increased morbidity. On the other hand, surgical resection should be as complete as possible to avoid the risk of future recurrence.

\section{Radiotherapy}

Becker et $a l^{18}$ reported a series of 24 secondary optic nerve sheath meningiomas undergoing stereotactic fractionated radiotherapy (SFRT) using $6 \mathrm{MV}$ photons from linear accelerators. The median total tumour dose was 54 Gy using $1.8 \mathrm{~Gy} /$ fraction. The visual fields were improved in six of 24 examined eyes and visual acuity in seven of 26 in this series. Stable visual fields and stable visual acuity were observed in 17 of 24 and 19 of 26 patients, respectively, without toxicity. Secondary optic nerve sheath meningiomas were defined as arising from the intracranial meninges and subsequently involving the visual pathways. This means that they included all kinds of skull base pathology such as medial sphenoid wing meningiomas, cavernous sinus meningiomas, planum sphenoidale meningiomas, and perhaps also some tuberculum sellae meningiomas.

Other large series of cases receiving radiotherapy for skull base meningiomas have emphasised the stabilisation of the tumour volume with good clinical results. ${ }^{19-22}$ However, only one series-that of Pendl et $a^{21}$-included three sellar meningiomas. The high efficacy of SFRT for large skull base meningiomas and the long term results have also been reported by Debus et al. ${ }^{23}$ Their overall survival rate was $97 \%$ after five years and $96 \%$ after 10 years (WHO grade I). Local treatment failure was observed in three of 180 patients with WHO grade I tumours and in two of nine patients with grade II tumours. A volume reduction of $>50 \%$ was observed in $14 \%$. Pre-existing cranial nerve symptoms resolved completely in $28 \%$ and treatment induced toxicity occurred in $1.6 \%$.

We think that radiotherapy should be considered in elderly patients and in those with subtotally resected tumours. Nevertheless, surgery remains the treatment of choice in tuberculum sellae meningiomas.

\section{Conclusions}

The goals of surgery are tumour control, minimal morbidity, and visual improvement. The duration of preoperative symptoms has a strong influence on visual outcome. Thus early diagnosis is desirable for successful treatment with a better chance of good postoperative visual outcome. Tumour extension into the optic canal is quite common and required optic canal deroofing and gentle tumour removal.

\section{ACKNOWLEDGEMENTS}

We thank Professor Seeger for the professional drawing of the surgical anatomy of tuberculum sellae meningiomas.

\section{Authors' affiliations}

U Schick, W Hassler, Clinic of Neurological Surgery, Wedau Kliniken, Duisburg, Germany

Competing interests: none declared

\section{REFERENCES}

1 DeMonte F. Surgical treatment of anterior basal meningiomas. J Neurooncol 1996;29:239-48.

2 Mourits MP, van der Sprenkel JW. Orbital meningiomas, the Utrecht experience. Orbit 2001;20:25-33.

3 Arai $\mathrm{H}$, Sato K, Okuda O, et al. Transcranial transsphenoidal approach for tuberculum sellae meningiomas. Acta Neurochir (Wien) 2000;142:751-7.

4 Ohta K, Yasuo K, Morikawa M, et al. Treatment of tuberculum sellae meningiomas: a long-term follow-up study. J Clin Neurosci 2001;8:26-31. 
5 Fahlbusch R, Schott W. Pterional surgery of meningiomas of the tuberculum sellae and planum sphenoidale: surgical results with special consideration of ophthalmological and endocrinological outcomes. J Neurosurg 2002;96:235-43.

6 Raco A, Bristot R, Domenicucci $M$, et al. Meningiomas of the tuberculum sellae. Our experience in 69 cases surgically treated between 1973 and 1993. J Neurosurg Sci 1999;43:253-60.

7 Al-Mefty O, Holoubi A, Rifai A, et al. Microsurgical removal of suprasellar meningiomas. Neurosurgery 1985;16:364-72.

8 Hassler WE, Eggert $\mathrm{H}$. Extradural and intradural microsurgical approaches to lesions of the optic canal and the superior orbital fissure. Acta Neurochir (Wien) 1985;74:87-93.

9 Yasargil MG. Anterior cranial fossa meningiomas. In: Yasargil MG, eds. Microneurosurgery, vol IVB. Stuttgart: Thieme Verlag, 1996:141-2.

10 Hollenhorst RW, Younge BR. Ocular manifestations produced by adenoma of the pituitary gland: analysis of 1000 cases. In: Kohler PO, Ross GT, eds. Diagnosis and treatment of pituitary tumors. New York: American Elsevier Publishing Co, 1973:53.

11 Symon L, Rosenstein J. Surgical management of suprasellar meningioma. The influence of tumor size, duration symptoms and microsurgery on surgical outcome in 101 patients. J Neurosurg 1984;61:633-41.

12 Zevgaridis D, Medele RJ, Muller A, et al. Meningiomas of the sellar region presenting with visual impairment: impact of various prognostic factors on surgical outcome in 62 patients. Acta Neurochir (Wien) 2001:143:471-6.

13 Mathiesen T, Lindquist C, Kihlstrom L, et al. Recurrence of cranial base meningiomas. Neurosurgery 1996;39:2-9.
14 Cornu P, Chatelier G, Dagreou F. Intracranial meningiomas in elderly patients: postoperative morbidity and mortality. Factors predictive of outcome. Acta Neurochir (Wien) 1990;102:98-102.

15 Shimano H, Nagasawa S, Kawabata S, et al. Surgical strategy for meningioma extension into the optic canal. Neurol Med Chir (Tokyo) 2000;40:447-52.

16 Andrews D, Wilson CB. Suprasellar meningiomas: the effect of tumor location on postoperative visual outcome. J Neurosurg 1988;69:523-8.

17 Lee JH, Jeun SS, Evans J, et al. Surgical management of clinoidal meningiomas. Neurosurgery 2001:48:1012-17.

18 Becker G, Jeremic B, Pitz S, et al. Stereotactic fractionated radiotherapy in patients with optic nerve sheath meningioma. Int J Radiat Oncol Biol Phys 2002;54:1422-9

19 Chang JH, Chang JW, Choi JY, et al. Complications after gamma knife radiosurgery for benign meningiomas. I Neurol Neurosurg Psychiatry 2003;74:226-30

20 Mendenhall WM, Morris CG, Amdur RJ, et al. Radiotherapy alone or after subtotal resection for benign skull base meningiomas. Cancer 2003;98:1473-82.

21 Pendl G, Eustacchio S, Unger F. Radiosurgery as alternative treatment for skull base meningiomas. J Clin Neurosci 2001;8(suppl 1):12-14.

22 Villavicencio AT, Black PM, Shrieve DC, et al. Linac radiosurgery for skull base meningiomas. Acta Neurochir (Wien) 2001;143:1141-52.

23 Debus J, Wuendrich M, Pirzkall A. High efficacy of fractionated stereotactic radiotherapy of large base-of-skull meningiomas: long-term results. J Clin Oncol 2001; 19:3547-53

\section{NEUROLOGICAL PICTURE}

\section{Bilateral first rib fractures due to tardive dystonia} history of previous neuroleptic use was admitted for investigation of a 1 year history of dystonia. On admission he had severe axial and limb dystonia with opisthotonus. Following extensive investigation for other causes of dystonia, a diagnosis of tardive dystonia secondary to neuroleptic exposure was made.

A routine chest radiograph revealed bilateral first rib fractures (fig 1). Such fractures are extremely rare because the first ribs are deeply placed and well protected by the shoulder girdle, lower neck musculature, and clavicles, ${ }^{1}$ and almost always occur in the context of severe, generalized trauma. However, rare cases of non-traumatic first rib fractures caused by sudden violent contraction of the neck muscles or repeated muscular pulling, for example during sporting activities, have been described. ${ }^{2}$ Traumatic first rib fractures typically occur at the subclavian groove where the subclavian artery crosses the thinnest portion of the rib, often causing injury to adjacent vascular, pulmonary, or mediastinal structures. By contrast, non-traumatic fractures rarely injure adjacent structures and conservative management is indicated.

The patient reported here had no history of previous trauma, and had no abnormal physical signs in the chest, nor any distal neurovascular deficit in the arms. We propose that the bilateral first rib fractures in this case resulted from tension on the scalene muscles during repetitive, violent opisthotonic spasms. To our knowledge, this is a unique neurological cause of bilateral first rib fractures.

D Hacking, D J Werring

The National Hospital for Neurology and Neurosurgery, Queen Square, London WCIN 3BG, United Kingdom

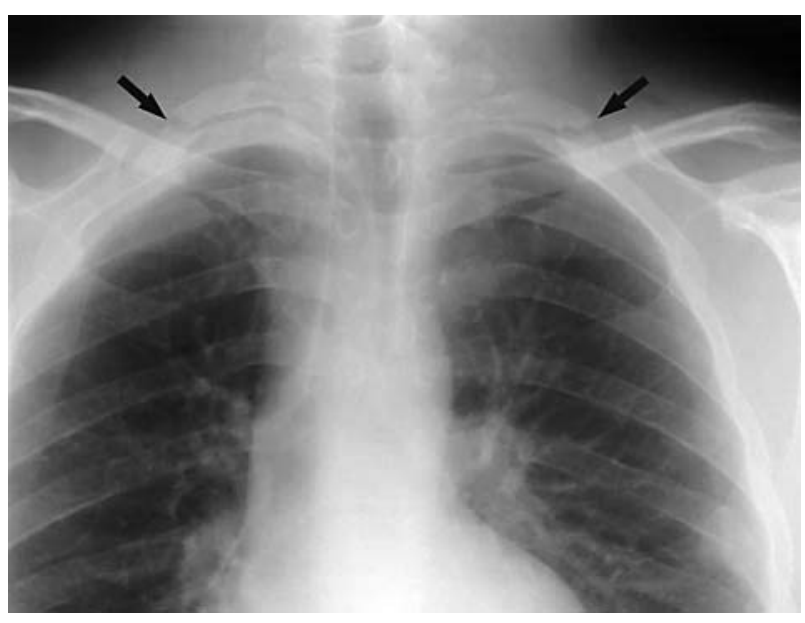

Figure 1 Chest radiograph showing bilateral first rib fractures (arrowed)

\section{References}

1 McAdam JG, Templeton JL, Nixon JR. Isolated fractures of the first ribs - an indication of major cervico mediastinal injury. Injury 1986;17:226-7.

2 Vikramaditya, Pritty P. Two cases of isolated first rib fracture. Emerg Med J 2001;18:498-9. 